\title{
PERDA DE CARGA EM MICROTUBOS E CONECTORES UTILIZADOS EM MICROASPERSÃO
}

\section{DANIELI B. ZITTERELL ${ }^{1}$, JOSÉ A. FRIZZONE ${ }^{2}$, OSVALDO RETTORE NETO ${ }^{3}$, MARIO N. ULLMANN ${ }^{4}$}

\begin{abstract}
RESUMO: Este trabalho foi desenvolvido com o objetivo de ajustar equações que estimam a perda distribuída de carga em microtubos utilizados em microaspersão e a perda localizada de carga na passagem lateral do fluxo por meio dos conectores na linha lateral. A perda distribuída de carga foi determinada em quatro diâmetros de microtubos com nove a dez repetições para 15 vazões, por meio da aplicação do teorema de Bernoulli. $O$ fator de atrito (f) foi estimado fixando-se o valor de $\mathrm{m}=0,25$ e calibrando-se o valor do parâmetro c (0,290). A perda localizada de carga foi determinada por diferença entre perda de carga no microtubo mais conector e perda de carga no microtubo. Dois modelos de conectores foram utilizados e caracterizados quanto ao diâmetro interno e dimensões. Uma aproximação matemática foi proposta para calcular a perda localizada de carga com base em coeficiente de carga cinética do conector (K'), que leva em consideração as dimensões do conector e do microtubo e independência das forças viscosas para $\operatorname{Re}>5.000$. As variações de vazão e de pressão entre os emissores situados nos extremos da linha lateral mostraram-se sensíveis à perda de carga na passagem lateral pelo conector mais a perda de carga no microtubo.
\end{abstract}

PALAVRAS-CHAVE: perda localizada de carga, perda distribuída de carga, comprimento equivalente, coeficiente de carga cinética.

\section{HEAD LOSS IN MICROTUBES AND CONNECTORS USED IN MICROSPRINKLER SYSTEMS}

\begin{abstract}
This work was carried out aimed at presenting equations to estimate the continuous head loss in microtubes and the local head loss in the connector used on microsprinklers lateral lines. The continuous head loss was determined using Bernoulli's theorem for four microtubes diameters, each one with nine to ten replications for 15 flowrates. The Darcy-Weisbach friction factor was estimated by setting $m$ to 0.25 and by calibrating the parameter value $c$ to 0.290 . The local head loss was determined by subtracting the head loss on the connector and microtube from the head loss on the microtube. Two types of connectors were used and characterized by its internal diameters and its dimensions. A mathematical approach to compute the local head loss was proposed based on a kinetic head connector coefficient $\left(K^{\prime}\right)$. This coefficient is a function of connector and microtube dimensions and independent of viscous forces up to $\operatorname{Re}>5,000$. Flowrate and pressure at emitters located at the far end of the lateral line were sensitive the head losses at the connectors and in the microtube.
\end{abstract}

KEYWORDS: local head loss, continuous head loss, equivalent length, kinetic head coefficient.

\footnotetext{
${ }^{1}$ Eng ${ }^{\mathrm{a}}$ Agrônoma, Mestranda em Irrigação e Drenagem, Departamento de Engenharia Rural, ESALQ/USP, Piracicaba - SP, Fone: (0XX19) 3447-8574, danieli@usp.br

${ }^{2}$ Eng $^{\mathrm{O}}$ Agrônomo, Prof. Titular, Departamento de Engenharia Rural, ESALQ/USP, Piracicaba - SP.

${ }^{3}$ Eng $^{0}$ Agrícola, M.Sc. em Irrigação e Drenagem, Departamento de Engenharia Rural, ESALQ/USP, Piracicaba - SP.

${ }^{4}$ Eng ${ }^{0}$ Agrônomo, Prof. Titular, Departamento de Agronomia, CAV/UDESC, Lages - SC.

Recebido pelo Conselho Editorial em: 27-5-2009

Aprovado pelo Conselho Editorial em: 16-10-2009 


\section{INTRODUÇÃO}

Alta uniformidade de distribuição de água permite a otimização do uso da água, da energia e de nutrientes. Para garantir alta uniformidade, é indispensável um dimensionamento hidráulico adequado, exigindo que características hidráulicas dos seus componentes sejam conhecidas. Como a microirrigação conta com elevado número de tubulações e peças acessórias, a perda de carga em microtubos e conectores pode assumir valores consideráveis nos projetos desses sistemas.

Microtubos e conectores são componentes indispensáveis na montagem de um sistema de microaspersão. A perda de energia causada pelo microtubo é alta devido ao seu pequeno diâmetro interno, que pode variar de 2,8 a $7 \mathrm{~mm}$. Uma perda adicional é causada pela presença do conector que, ao ocupar área interna da seção do microtubo, modifica as linhas de fluxo e causa turbulência.

Diversas relações matemáticas são utilizadas nos cálculos de perda de carga. Dentre elas, a equação de Darcy-Weisbach que é uma das mais completas para o cálculo da perda de carga em tubulações, já que envolve todas as variáveis responsáveis pelo fenômeno. Sua fórmula é representada pela eq.(1), em que o fator de atrito $f$ varia de acordo com a condição de escoamento e deve ser determinado por meio de equações matemáticas para cada regime.

$$
\mathrm{hf}=\mathrm{f} \frac{\mathrm{L}}{\mathrm{D}} \frac{\mathrm{V}^{2}}{2 \mathrm{~g}}
$$

em que,

hf - perda de carga, m;

$\mathrm{f}$ - fator de atrito, adimensional;

$\mathrm{L}$ - comprimento do tubo, $\mathrm{m}$;

$\mathrm{V}$ - velocidade média de escoamento do fluido, $\mathrm{m} \mathrm{s}^{-1}$;

D - diâmetro interno do tubo, $\mathrm{m}$, e

$\mathrm{g}$ - aceleração da gravidade, $\mathrm{m} \mathrm{s}^{-2}$.

No regime laminar, o fator de atrito pode ser determinado pela equação de Hagen-Poiseuille ( $f=64 / R e$ ), dependente apenas do número de Reynolds $(\mathrm{Re})$. Para o regime turbulento, além do número de Reynolds, a estimativa do valor $\mathrm{f}$ depende da rugosidade relativa das paredes do tubo $(\varepsilon / \mathrm{D})$, em que $\varepsilon$ é a rugosidade absoluta do tubo.

No escoamento turbulento completo em tubos rugosos, f pode ser calculado pela equação de Colebrook-White (PORTO, 1998; ROMEO et al., 2002; YOO \& SINGH, 2005; SONNAD \& GOUDAR, 2006; CARDOSO et al., 2008). Essa é uma combinação das equações de Prandtl e de Von Karman, válida para $0 \leq \varepsilon / D \leq 0,05$ e $210^{3}<R e<10^{8}$. É válida também para o caso limite de tubos lisos $(\varepsilon \rightarrow 0)$ e escoamento completamente turbulento. Todavia, BERNUTH (1990) considera esse método ineficiente para o cálculo de perda de carga em tubos plásticos de pequenos diâmetros.

No escoamento turbulento completo em tubos lisos, o coeficiente $f$ independe da rugosidade do conduto, já que o tamanho das asperezas não influi na turbulência do escoamento. Nesse caso, a equação de Von Karman (PORTO, 1998) pode ser utilizada para Re entre $410^{3}$ e 3,4 106.

No regime turbulento em tubos lisos, Blasius propôs a eq.(2) fundamentada nas leis da similaridade, que é dada em função apenas do número de Reynolds, válida para $410^{3} \leq \operatorname{Re} \leq 10^{5}$.

$$
\mathrm{f}=\frac{\mathrm{c}}{\operatorname{Re}^{\mathrm{m}}}
$$

em que, c e m são os coeficientes de Blasius, 0,316 e 0,25, respectivamente.

De acordo com ALVES \& PORTO (2002) e BERNUTH (1990), a equação de Blasius é válida para tubos hidraulicamente lisos desde que Reynolds não ultrapasse $10^{5}$. Quando Re é muito elevado, a precisão é reduzida, pois o fator de atrito se aproxima de zero. Quando $\operatorname{Re}<410^{3}$, em regime laminar ou zonas críticas, a equação de Blasius superestima o fator de atrito. Para o 
escoamento na zona de transição, $210^{3}<\operatorname{Re}<310^{3}$, o fator de atrito pode ser aproximado por um valor constante, igual a 0,04 (YITAYEW, 2009).

Para JUANA et al. (2002), a introdução do fator de atrito de Blasius na equação de DarcyWeisbach promove uma estimativa precisa das perdas de carga em regimes turbulentos com baixa rugosidade para $310^{3} \leq \operatorname{Re} \leq 10^{5}$. BERNUTH (1990) comenta que a combinação dessas duas equações resulta em uma equação dimensionalmente homogênea (ambas possuem bases teóricas), exata para tubos plásticos quando $\operatorname{Re} \leq 10^{5}$, e adequada para tubos de pequenos diâmetros, já que Re nesse intervalo não é restritivo em sistemas com diâmetros inferiores a $80 \mathrm{~mm}$. Além disso, mudanças de viscosidade podem ser facilmente corrigidas por meio de fatores disponíveis em tabelas.

A presença do conector no microtubo provoca perda localizada adicional devido à ocupação de uma área interna da seção do microtubo que modifica as linhas de fluxo e causa turbulência. Essas perdas são comumente desconsideradas nos projetos e, como consequência, obtêm-se diâmetros e comprimentos de linhas errôneos (YILDIRIM, 1997).

A perda localizada de carga é expressa classicamente pela variação da energia cinética multiplicada por um fator $\mathrm{K}$ [eq. (3)]:

$$
\mathrm{hf}_{\mathrm{L}}=\mathrm{K} \frac{\mathrm{V}^{2}}{2 \mathrm{~g}}
$$

em que,

$\mathrm{hf}_{\mathrm{L}}$ - perda de carga localizada, $\mathrm{m}, \mathrm{e}$

$\mathrm{K}$ - coeficiente de carga cinética ou de resistência de perfil, adimensional.

$\mathrm{O}$ coeficiente $\mathrm{K}$ depende das características geométricas da peça e do número de Reynolds, reduzindo com o aumento de $\mathrm{Re}$ até certo limite a partir do qual se mantém constante (BAGARELLO et al., 1997; PROVENZANO \& PUMO, 2004; JUANA et al., 2002 e CARDOSO \& FRIZZONE, 2007).

O presente trabalho tem como objetivo ajustar equações que estimam a perda distribuída de carga em microtubos de diferentes diâmetros, bem como a perda localizada de carga provocada pela passagem lateral do fluxo em conectores de microaspersores com a linha lateral.

\section{MATERIAL E MÉTODOS}

O presente estudo foi conduzido no Laboratório de Irrigação do Departamento de Engenharia Rural da Escola Superior de Agricultura "Luiz de Queiroz" - USP, Piracicaba - SP. A bancada de testes e seus elementos estão esquematizados na Figura 1. Com o objetivo de evitar variações de vazão, o conjunto motobomba foi ligado a um inversor de freqüência, que manteve sua rotação constante. Diferentes vazões foram obtidas pelo controle dos registros. As pressões na entrada do microtubo foram monitoradas por manômetro digital (escala 0 - $1.500 \mathrm{kPa}$ ) com fundo de escala de um dígito decimal.

Para a determinação da perda distribuída de carga no microtubo, um segmento de tubo de PVC de $50 \mathrm{~mm}$ de diâmetro e $1 \mathrm{~m}$ de comprimento com final fechado foi instalado. Essa linha possuía uma tomada de pressão e um orifício, em que o microtubo foi inserido e fixado com resina epoxi.

A seção de escoamento dos microtubos foi determinada em 20 segmentos em forma de anéis, medindo-se o diâmetro e a espessura das paredes em um projetor óptico de perfil, marca Starrett, modelo HB 400. Na Tabela 1, apresentam-se as medidas de diâmetro e de espessura da parede de cada modelo de microtubo estudado. 


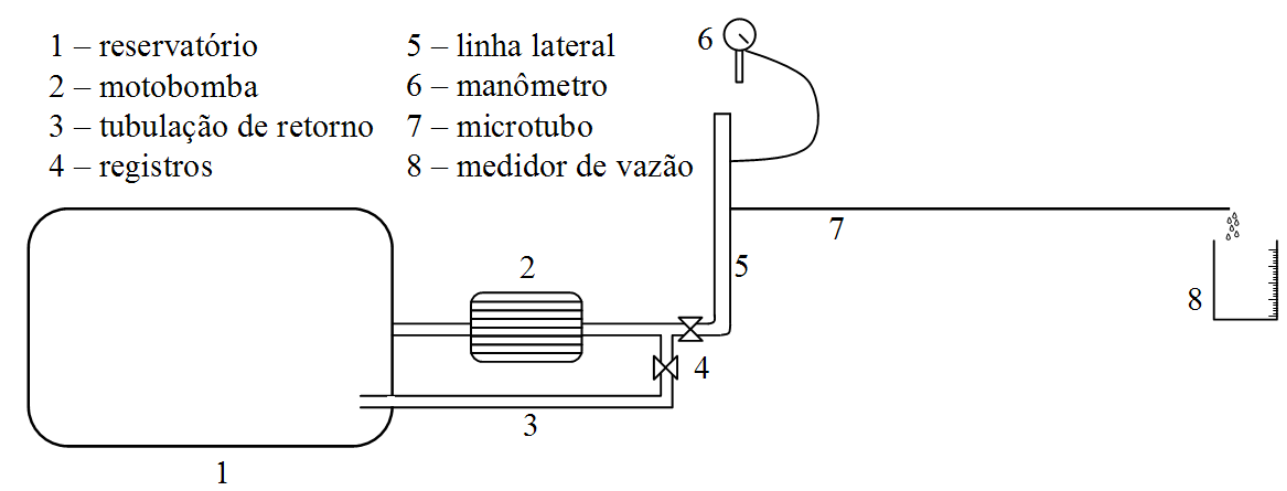

FIGURA 1. Esquema da instalação utilizada na determinação da perda de carga. Layout of the experimental installation for head loss determination.

Para a determinação da perda distribuída de carga no microtubo, um segmento de tubo de PVC de $50 \mathrm{~mm}$ de diâmetro e $1 \mathrm{~m}$ de comprimento com final fechado foi instalado. Essa linha possuía uma tomada de pressão e um orifício, em que o microtubo foi inserido e fixado com resina epoxi.

A seção de escoamento dos microtubos foi determinada em 20 segmentos em forma de anéis, medindo-se o diâmetro e a espessura das paredes em um projetor óptico de perfil, marca Starrett, modelo HB 400. Na Tabela 1, apresentam-se as medidas de diâmetro e de espessura da parede de cada modelo de microtubo estudado.

Os ensaios foram realizados de modo a medir a pressão na entrada do microtubo e a vazão ao final. A perda de carga no microtubo foi determinada por meio da aplicação do teorema de Bernoulli entre as seções de entrada e de saída do tubo (1 e 2, respectivamente):

$$
\mathrm{Z}_{1}+\frac{\mathrm{P}_{1}}{\gamma}+\frac{\mathrm{V}_{1}^{2}}{2 \mathrm{~g}}=\mathrm{Z}_{2}+\frac{\mathrm{P}_{2}}{\gamma}+\frac{\mathrm{V}_{2}^{2}}{2 \mathrm{~g}}+\mathrm{hf}
$$

em que,

$\mathrm{Z}$ - carga de posição, $\mathrm{m}$;

$\mathrm{P} / \gamma$ - carga de pressão, $\mathrm{m}$, e

$\mathrm{V}_{2} / 2 \mathrm{~g}$ - carga de velocidade, $\mathrm{m}$.

TABELA 1. Características dos microtubos utilizados no ensaio. Characteristics of microtubes used in the experiments.

\begin{tabular}{cccccccc}
\hline \multirow{2}{*}{ Modelo } & \multicolumn{3}{c}{ Diâmetro Interno (mm) } & & \multicolumn{3}{c}{ Espessura da Parede (mm) } \\
\cline { 2 - 3 } & $\begin{array}{c}\text { Média } \\
(\mathrm{mm})\end{array}$ & $\begin{array}{c}\text { Desvio- } \\
\text {-Padrão }(\mathrm{mm})\end{array}$ & $\begin{array}{c}\text { Coeficiente de } \\
\text { Variação }(\%)\end{array}$ & & $\begin{array}{c}\text { Média } \\
(\mathrm{mm})\end{array}$ & $\begin{array}{c}\text { Desvio- } \\
\text {-Padrão (mm) }\end{array}$ & $\begin{array}{c}\text { Coeficiente de } \\
\text { Variação (\%) }\end{array}$ \\
\hline PE 4,0 7,0 & 4,47 & 0,0290 & 0,659 & & 1,29 & 0,0380 & 2,95 \\
PE 5,6 8,0 & 5,66 & 0,0180 & 0,318 & & 1,22 & 0,0290 & 2,40 \\
PE 4,0 7,0 & 4,09 & 0,0290 & 0,709 & & 1,39 & 0,0560 & 4,03 \\
PE 4,5 7,0 & 4,63 & 0,0460 & 0,994 & & 1,22 & 0,0550 & 4,51 \\
\hline
\end{tabular}

As cargas de posição $\left(Z_{1}\right.$ e $\left.Z_{2}\right)$ são componentes nulas neste ensaio, pois o microtubo estava em nível. A carga de pressão na alimentação do microtubo $\left(\mathrm{P}_{1} / \gamma\right)$ foi obtida pela leitura no manômetro conectado ao tubo de derivação. Já a carga de pressão no final do microtubo $\left(\mathrm{P}_{2} / \gamma\right)$ é nula, pois a água escoava livremente para a atmosfera. A carga de velocidade $\left(\mathrm{V}_{1}^{2} / 2 \mathrm{~g}\right)$ na entrada do microtubo foi desconsiderada, assumindo que não houve componente de velocidade na direção do microtubo no seu ponto de inserção. A carga de velocidade $\left(\mathrm{V}_{2}^{2} / 2 \mathrm{~g}\right)$ ao final do tubo foi determinada por meio da vazão, sendo essa a razão entre o volume de água coletado num recipiente 
obtido por gravimetria e o tempo de coleta. Com base nesses pressupostos, a perda de carga do microtubo pode ser representada pela eq.(5):

$$
\mathrm{hf}=\frac{\mathrm{P}_{1}}{\gamma}-\frac{\mathrm{V}_{2}^{2}}{2 \mathrm{~g}}
$$

Cada repetição do ensaio consistiu de um segmento de $20 \mathrm{~m}$ de microtubo. Para os microtubos de 4,47; 4,09 e 4,63 mm, nove segmentos foram ensaiados, e para o microtubo de 5,66 $\mathrm{mm}$, dez segmentos. Cada segmento de microtubo foi posto em funcionamento com 15 pressões de operação, que variaram de $294 \mathrm{kPa}(30 \mathrm{~m})$ até aproximadamente $39,2 \mathrm{kPa}(4 \mathrm{~m})$, ou até que o número de Reynolds fosse superior a $310^{3}$. Considerou-se Re maior que $310^{3}$ como regime turbulento com o intuito de evitar as incertezas de representação do fenômeno de perda de carga em regime de transição. A temperatura da água foi monitorada durante os ensaios para corrigir a viscosidade cinemática nos cálculos da perda de carga.

Os dados coletados possibilitaram o ajuste de uma equação potencial na forma da eq.(6), relacionado a perda de carga com a vazão para cada diâmetro de microtubo.

$$
\mathrm{hf}_{\mathrm{m}}=\mathrm{kQ}^{\mathrm{n}}
$$

em que,

$\mathrm{hf}_{\mathrm{m}}$ - perda de carga (m) no microtubo com $20 \mathrm{~m}$ de comprimento;

$\mathrm{Q}$ - vazão, $\mathrm{m}^{3} \mathrm{~s}^{-1}, \mathrm{e}$

$\mathrm{k}$ e n - parâmetros, adimensional.

Os valores experimentais de perda de carga nos microtubos foram utilizados para calcular o fator de atrito da equação de Darcy-Weisbach [eq.(1)]. Explicitou-se f em função de hf, L, D e $\mathrm{V}^{2} / 2 \mathrm{~g}$. O coeficiente $\mathrm{c}$ foi calculado para $\mathrm{m}=0,25$ por meio de regressão linear entre $\mathrm{f}$ e $\mathrm{Re}^{-0,25}$.

Para a determinação da perda localizada de carga, considerou-se a condição em que o diâmetro do conector é inferior ao diâmetro do microtubo. Foram utilizados dois modelos de conectores que foram caracterizados quanto à área de protrusão, diâmetro interno e dimensões, como mostra a representação esquemática das Figuras 2 e 3. As medições foram efetuadas no projetor óptico de perfil em 15 conectores de cada modelo, sendo suas características apresentadas na Tabela 2.

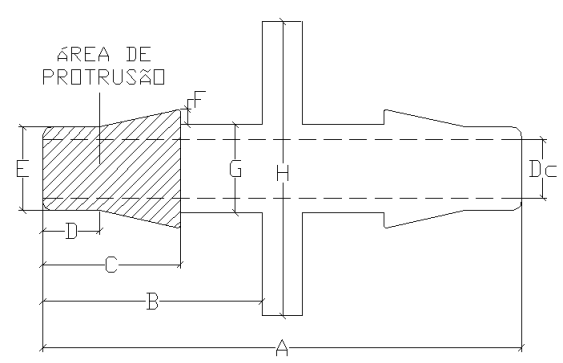

FIGURA 2. Representação esquemática das dimensões do conector de 2,95 $\mathrm{mm}$. Schematic representation of the dimensions of $2.95 \mathrm{~mm}$ connector.

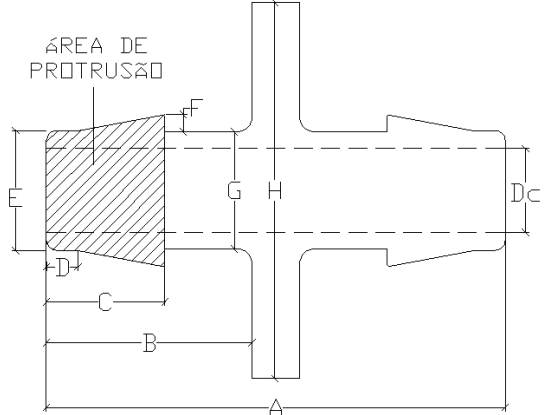

FIGURA 3. Representação esquemática das dimensões do conector de $3,91 \mathrm{~mm}$. Schematic representation of the dimensions of $3.91 \mathrm{~mm}$ connector.

Os microtubos de diâmetros internos 4,47; 4,09 e 4,63 mm foram ensaiados com o conector de diâmetro 2,95 mm. O microtubo de diâmetro interno 5,66 mm adaptou-se ao conector de diâmetro 3,91 mm. Os conjuntos microtubo/conector foram inseridos em linha de derivação de polietileno de $25 \mathrm{~mm}$ de diâmetro, 1,0 m de comprimento e com final fechado. Distante $15 \mathrm{~cm}$ da inserção, foi instalada uma tomada de pressão. 
TABELA 2. Características dos conectores utilizados no ensaio. Characteristics of connectors used in the experiments.

\begin{tabular}{cccccccc}
\hline Características & \multicolumn{3}{c}{$2,95 \mathrm{~mm}$} & & \multicolumn{3}{c}{$3,91 \mathrm{~mm}$} \\
\cline { 2 - 3 } \cline { 6 - 7 } Geométricas & Média & Desvio & C.V. $(\%)$ & & Média & Desvio & C.V. $(\%)$ \\
\hline Área de protrusão no tubo & $33,1 \mathrm{~mm}^{2}$ & $0,298 \mathrm{~mm}^{2}$ & 0,900 & & $33,8 \mathrm{~mm}^{2}$ & $0,281 \mathrm{~mm}^{2}$ & 0,831 \\
Diâmetro $\left(\mathrm{D}_{\mathrm{c}}\right)$ & $2,95 \mathrm{~mm}$ & $0,021 \mathrm{~mm}$ & 0,712 & & $3,91 \mathrm{~mm}$ & $0,028 \mathrm{~mm}$ & 0,716 \\
$\mathrm{~A}$ & $24,2 \mathrm{~mm}$ & $0,127 \mathrm{~mm}$ & 0,524 & & $21,5 \mathrm{~mm}$ & $0,041 \mathrm{~mm}$ & 0,191 \\
$\mathrm{~B}$ & $11,1 \mathrm{~mm}$ & $0,083 \mathrm{~mm}$ & 0,748 & & $9,63 \mathrm{~mm}$ & $0,037 \mathrm{~mm}$ & 0,384 \\
$\mathrm{C}$ & $6,96 \mathrm{~mm}$ & $0,041 \mathrm{~mm}$ & 0,589 & & $5,54 \mathrm{~mm}$ & $0,025 \mathrm{~mm}$ & 0,451 \\
$\mathrm{D}$ & $2,88 \mathrm{~mm}$ & $0,054 \mathrm{~mm}$ & 1,88 & & $1,48 \mathrm{~mm}$ & $0,073 \mathrm{~mm}$ & 4,930 \\
$\mathrm{E}$ & $4,20 \mathrm{~mm}$ & $0,028 \mathrm{~mm}$ & 0,667 & & $5,59 \mathrm{~mm}$ & $0,016 \mathrm{~mm}$ & 0,286 \\
$\mathrm{~F}$ & $0,78 \mathrm{~mm}$ & $0,086 \mathrm{~mm}$ & 11,0 & & $0,815 \mathrm{~mm}$ & $0,067 \mathrm{~mm}$ & 8,220 \\
$\mathrm{G}$ & $4,44 \mathrm{~mm}$ & $0,089 \mathrm{~mm}$ & 2,01 & & $5,47 \mathrm{~mm}$ & $0,030 \mathrm{~mm}$ & 0,548 \\
$\mathrm{H}$ & $14,8 \mathrm{~mm}$ & $0,365 \mathrm{~mm}$ & 2,47 & & $17,6 \mathrm{~mm}$ & $0,288 \mathrm{~mm}$ & 1,640 \\
\hline
\end{tabular}

A perda de carga no conjunto microtubo mais conector foi obtida com base na eq.(5), medindo-se a pressão na entrada do microtubo e a vazão ao final. Uma equação potencial relacionando a perda de carga com a vazão para cada diâmetro [eq.(7)] foi ajustada:

$$
\mathrm{hf}_{\mathrm{m}+\mathrm{c}}=\mathrm{kQ}^{\mathrm{n}}
$$

em que,

$\mathrm{hf}_{\mathrm{m}+\mathrm{c}}$ - perda de carga no microtubo mais conector, $\mathrm{m}$.

A perda de carga no conector foi determinada em função da diferença entre a perda de carga no microtubo mais conector e a perda de carga do microtubo sem conector, calculada pela eq.(8):

$$
\mathrm{hf}_{\mathrm{c}}=\mathrm{hf}_{\mathrm{m}+\mathrm{c}}-\mathrm{hf}_{\mathrm{m}}
$$

em que,

$\mathrm{hf}_{\mathrm{c}}$ - perda de carga no conector, $\mathrm{m}$.

Esse procedimento foi executado arbitrando-se vazões e calculando as referidas perdas de carga pelas eqs.(6) e (7). Esse artifício foi utilizado uma vez que é impraticável repetir os mesmos valores de vazão para os testes no microtubo mais conector e microtubo sem conector. Uma nova equação foi ajustada por análise de regressão utilizando-se de modelo potencial, dada pela eq.(9):

$$
\mathrm{hf}_{\mathrm{c}}=\mathrm{kQ}^{\mathrm{n}}
$$

A perda localizada de carga ocorre devido à dissipação de energia causada pela turbulência, sendo expressa em função da carga cinética corrigida por um coeficiente K, conforme a eq.(3). No modelo proposto [eq.(11)], optou-se por expressar essa perda de carga na forma de perda de carga unitária $\left(\mathrm{J}_{\mathrm{c}}\right)$, já que os conectores possuem comprimentos diferentes $\left(\mathrm{L}_{\mathrm{c}}\right)$, possibilitando o ajuste de um único coeficiente $\mathrm{K}^{\prime}$ por meio de regressão linear entre $\mathrm{V}_{\mathrm{c}}{ }^{2} / 2 \mathrm{~g}$ e $\mathrm{J}_{\mathrm{c}}$.

$$
\begin{aligned}
& \mathrm{hf}_{\mathrm{c}}=\mathrm{K}_{\mathrm{c}} \frac{\mathrm{V}_{\mathrm{c}}^{2}}{2 \mathrm{~g}} \frac{\mathrm{L}_{\mathrm{c}}}{\mathrm{D}_{\mathrm{c}}}=\frac{\mathrm{K}_{\mathrm{c}}}{\mathrm{D}_{\mathrm{c}}} \frac{\mathrm{V}_{\mathrm{c}}^{2}}{2 \mathrm{~g}} \mathrm{~L}_{\mathrm{c}}=\mathrm{K}^{\prime} \frac{\mathrm{V}_{\mathrm{c}}^{2}}{2 \mathrm{~g}} \mathrm{~L}_{\mathrm{c}} \\
& \mathrm{J}_{\mathrm{c}}=\frac{\mathrm{hf}_{\mathrm{c}}}{\mathrm{L}_{\mathrm{c}}}=\mathrm{K}^{\prime} \frac{\mathrm{V}_{\mathrm{c}}^{2}}{2 \mathrm{~g}}
\end{aligned}
$$

em que,

$\mathrm{K}_{\mathrm{c}}$ - fator de atrito ,adimensional;

$\mathrm{V}_{\mathrm{c}}$ - velocidade de escoamento no conector, $\mathrm{m} \mathrm{s}^{-1}$;

$\mathrm{L}_{\mathrm{c}}$ - comprimento do conector, $\mathrm{m}$;

$\mathrm{D}_{\mathrm{c}}$ - diâmetro interno do conector, $\mathrm{m}$;

$K^{\prime}$ - coeficiente de perda localizada de carga do conector $\left(K^{\prime}=K_{c} / D_{c}\right)$, e

$\mathrm{J}_{\mathrm{c}}$ - perda de carga unitária no conector, $\mathrm{m} \mathrm{m}^{-1}$. 
A perda localizada de carga no conector foi expressa também na forma de comprimento equivalente de tubulação, rearranjando os termos da equação de Darcy-Weisbach [eq.(1)] e da perda localizada de carga [eq.(11)], explicitando Le:

$$
\mathrm{Le}=\frac{\mathrm{K}^{\prime} \mathrm{L}_{\mathrm{c}} \mathrm{D}_{\mathrm{m}}^{5,25}}{\mathrm{v}^{0,25} \mathrm{cD}_{\mathrm{c}}^{4}} \mathrm{~V}_{\mathrm{m}}^{0,25}
$$

em que,

Le - comprimento equivalente de tubulação, m;

$\mathrm{D}_{\mathrm{m}}$ - diâmetro interno do microtubo, $\mathrm{m}$;

$\mathrm{V}_{\mathrm{m}}$ - velocidade de escoamento no microtubo, $\mathrm{m} \mathrm{s}^{-1}$, e

$v$ - viscosidade cinemática da água, $\mathrm{m}^{2} \mathrm{~s}^{-1}$.

\section{RESULTADOS E DISCUSSÃO}

\section{Perda distribuída de carga no microtubo}

Na Figura 4, apresentam-se as perdas de carga observadas experimentalmente em função da vazão nos quatro diâmetros de microtubos estudados. As equações ajustadas podem ser observadas na Tabela 3. Para todos os diâmetros, as equações apresentaram coeficiente de determinação superior a 0,999 para o modelo potencial. O expoente da vazão variou entre 1,78 e 1,80. De acordo com STREETER \& WYLIE (1982), citado por CAIXETA (1991), as perdas de carga são proporcionais às potências de 1,7 a 2,0 da velocidade média. De modo semelhante, o expoente da equação de Darcy Weisbach com $\mathrm{f}$ calculado pela equação de Blasius é igual a 1,75. Esses valores caracterizam o regime de escoamento turbulento e são muito próximos dos valores encontrados neste trabalho.
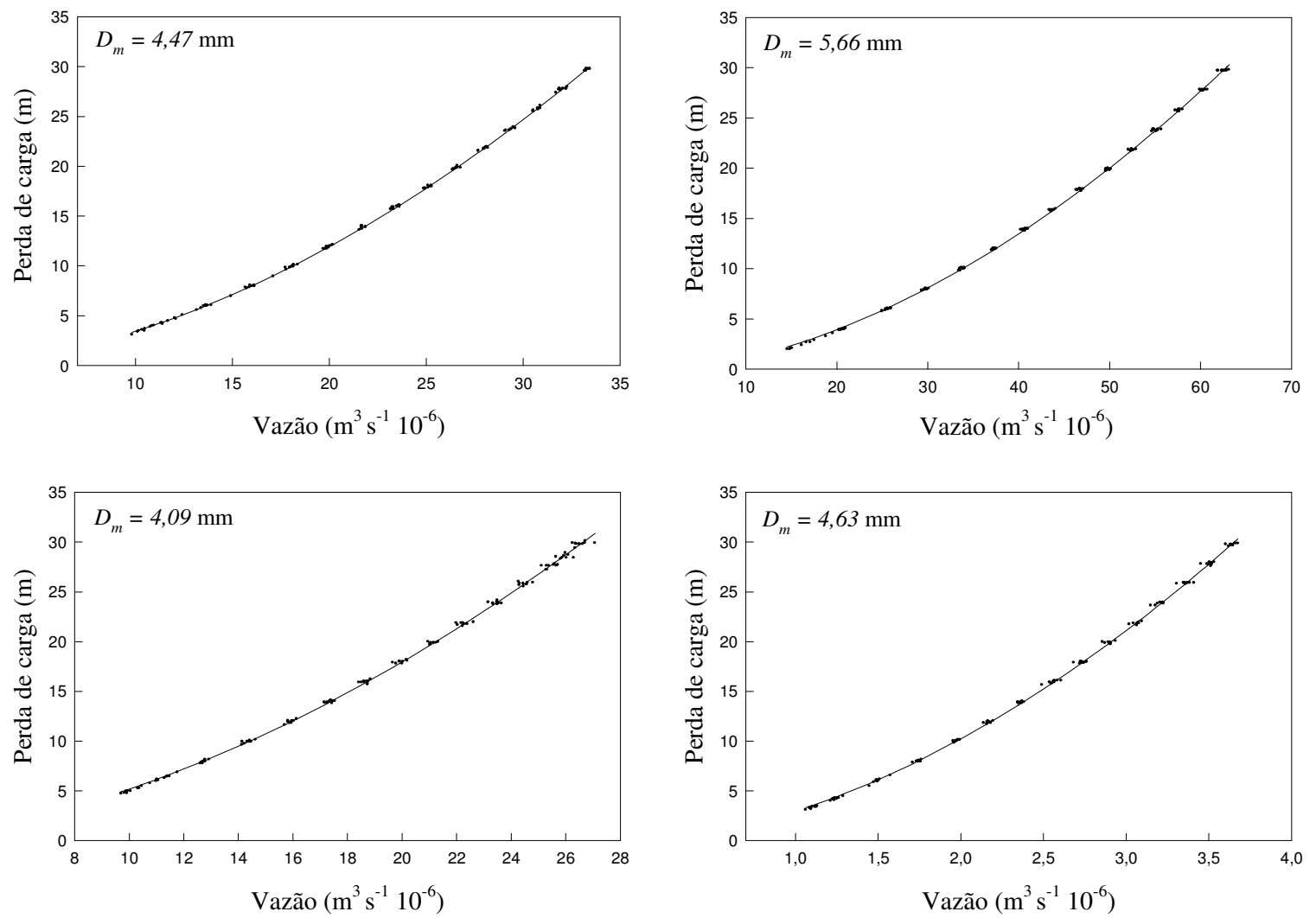

FIGURA 4. Perda de carga unitária (m) em $20 \mathrm{~m}$ de microtubo em função da vazão $\left(\mathrm{m}^{3} \mathrm{~s}^{-1}\right)$. Friction head loss $(\mathrm{m})$ in $20 \mathrm{~m}$ length microtube as a function of the discharge $\left(\mathrm{m}^{3} \mathrm{~s}^{-1}\right)$. 
TABELA 3. Equações de perda de carga (m) em $20 \mathrm{~m}$ de microtubo em função da vazão $\left(\mathrm{m}^{3} \mathrm{~s}^{-1}\right)$ para os microtubos estudados. Friction head loss $(\mathrm{m})$ in $20 \mathrm{~m}$ legth equations as a function of the discharge $\left(\mathrm{m}^{3} \mathrm{~s}^{-1}\right)$ to the microtubes tested.

\begin{tabular}{ccc}
\hline $\mathrm{D}_{\mathrm{m}}(\mathrm{mm})$ & Equação de Perda de Carga Unitária & $\mathrm{R}^{\mathbf{2}}$ \\
\hline 4,47 & $\mathrm{hf}_{\mathrm{m}}=3,2310^{9} \mathrm{Q}^{1,80}$ & 0,999 \\
5,66 & $\mathrm{hf}_{\mathrm{m}}=8,7910^{8} \mathrm{Q}^{1,78}$ & 0,999 \\
4,09 & $\mathrm{hf}_{\mathrm{m}}=4,6710^{9} \mathrm{Q}^{1,79}$ & 0,999 \\
4,63 & $\mathrm{hf}_{\mathrm{m}}=2,4810^{9} \mathrm{Q}^{1,78}$ & 0,999 \\
\hline
\end{tabular}

\section{Coeficiente de atrito da equação universal}

Na Figura 5, apresenta-se a relação entre os fatores de atrito observados e $\operatorname{Re}^{-0,25}$, para $\operatorname{Re}$ entre 5.002 e 15.766. O coeficiente c $(0,290)$ foi obtido por meio de regressão linear para $\mathrm{m}=0,25$, com $\mathrm{R}^{2}=0,878$. BAGARELLO et al. (1995) encontraram $\mathrm{c}=0,302$ para $\mathrm{m}=0,25$ utilizando tubos de diâmetros 16; 20 e $25 \mathrm{~mm}$ e Re entre 3.037 e 31.373. CARDOSO et al. (2008) encontraram $\mathrm{c}=0,300$ para tubos de polietileno de baixa densidade de diâmetros $10 ; 13 ; 16,3 ; 17,4$ e $19,7 \mathrm{~mm}$, e Re entre $510^{3}$ e $6,810^{4}$, enquanto RETTORE NETO et al. (2009) encontrou para tubos de polietileno de diâmetro $15,5 \mathrm{~mm}$ e Re entre 8.244 e 35.127 o valor de 0,296 . A redução desses coeficientes comparando-se com o obtido inicialmente por Blasius $(0,316)$ pode ser explicada pelo aumento de diâmetro dos tubos de polietileno quando pressurizados, conforme apresentam FRIZZONE et al. (1998) e VILELA et al. (2003). BAGARELLO et al. (1995) atribuem esse efeito às melhorias no processo de fabricação dos tubos, sendo os atuais mais lisos do que os tubos utilizados por Blasius, quando definiu o modelo para cálculo do fator de atrito.

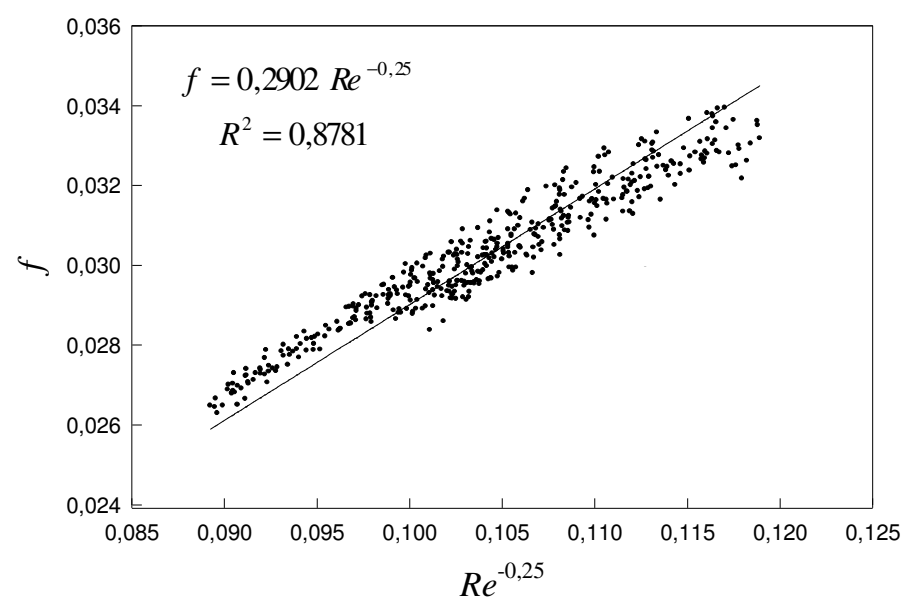

FIGURA 5. Relação entre fator de atrito $\mathrm{f}$ e $\mathrm{Re}^{-0,25}$ obtida pelo ajuste dos dados experimentais. Relationship between friction factor $f$ and $\operatorname{Re}^{-0,25}$ obtained by experimental data.

Utilizando-se c $=0,290$ e $\mathrm{m}=0,25$, a equação de perda de carga de Darcy-Weisbach para os microtubos estudados pode ser expressa na forma da eq.(13):

$$
\mathrm{J}_{\mathrm{m}}=0,0226 v^{0,25} \mathrm{Q}^{1,75} \mathrm{D}_{\mathrm{m}}^{-4,75}
$$

sendo, $\mathrm{J}_{\mathrm{m}}$ - perda de carga unitária no microtubo, $\mathrm{m} \mathrm{m}^{-1}$.

\section{Perda localizada de carga nos conectores}

Na Figura 6, apresenta-se o comportamento da perda localizada de carga na passagem lateral do fluxo por meio dos conectores. A curva contínua representa a perda de carga ajustada em $20 \mathrm{~m}$ de microtubo com conector e a curva tracejada é a perda de carga nesse mesmo trecho, porém sem a presença do conector. A diferença entre essas curvas é a perda de carga produzida por um conector. 
As equações ajustadas para as perdas de carga no microtubo mais conector e microtubo sem conector podem ser observadas na Tabela 4.
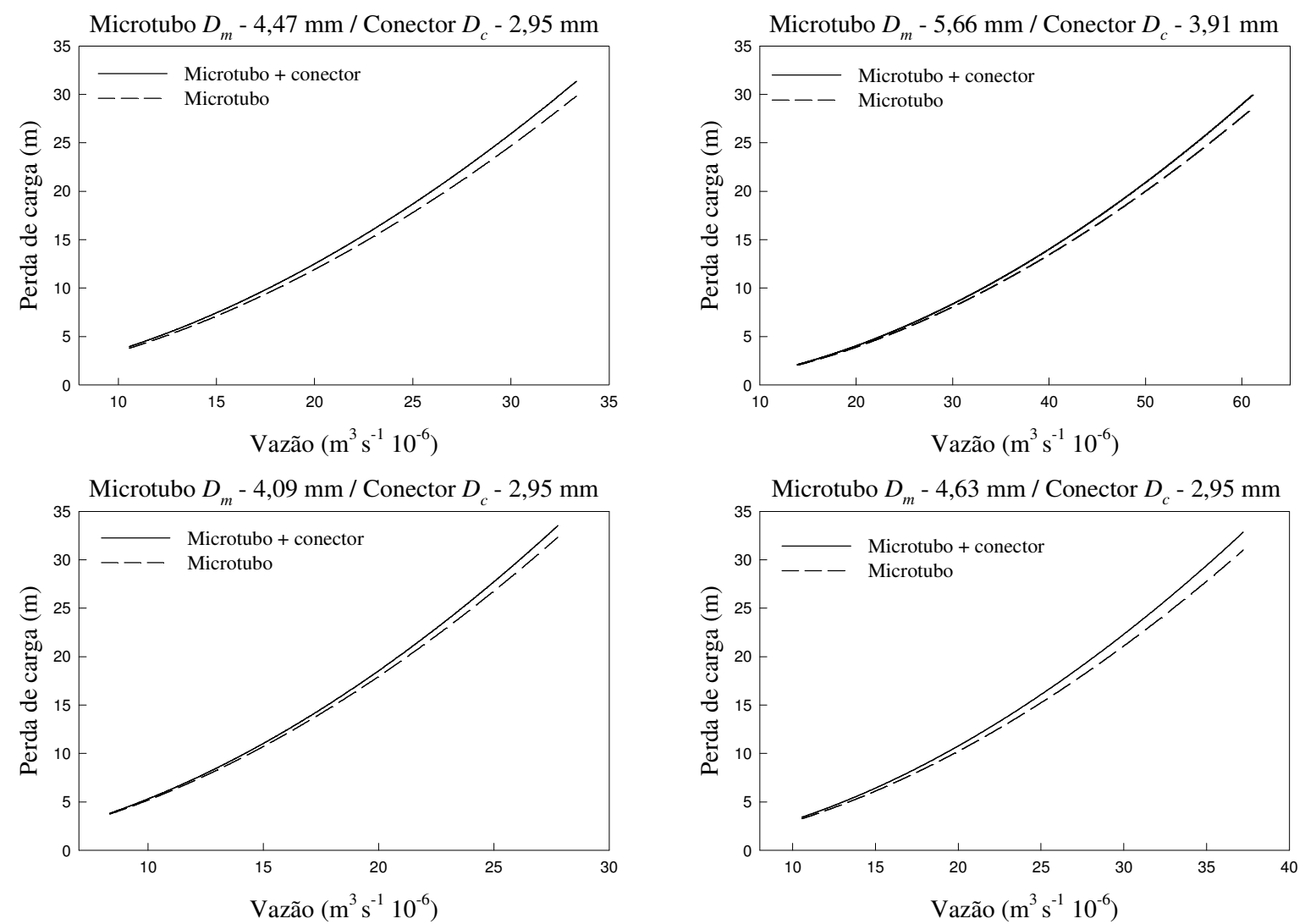

FIGURA 6. Perda de carga (m) em $20 \mathrm{~m}$ de tubulação em função da vazão $\left(\mathrm{m}^{3} \mathrm{~s}^{-1}\right)$ para o microtubo e microtubo mais conector. Friction head loss $(\mathrm{m})$ for a $20 \mathrm{~m}$ length pipe as a function of the discharge $\left(\mathrm{m}^{3} \mathrm{~s}^{-1}\right)$ for a microtube and microtube added connector.

TABELA 4. Equações de perda de carga (m) em função da vazão $\left(\mathrm{m}^{3} \mathrm{~s}^{-1}\right)$ para os microtubos com e sem conector. Head losses $(\mathrm{m})$ equations as a function of the discharge $\left(\mathrm{m}^{3} \mathrm{~s}^{-1}\right)$ for microtubes with/without connector.

\begin{tabular}{cccccc}
\hline $\begin{array}{c}\mathrm{D}_{\mathrm{m}} \\
(\mathrm{mm})\end{array}$ & $\begin{array}{c}\mathrm{D}_{\mathrm{c}} \\
(\mathrm{mm})\end{array}$ & $\begin{array}{c}\text { Perda de Carga no } \\
\text { Microtubo }\left(\mathrm{hf}_{\mathrm{m}}\right)\end{array}$ & $\mathrm{R}^{2}$ & $\begin{array}{c}\text { Perda de Carga no } \\
\text { Microtubo + Conector }\left(\mathrm{hf}_{\mathrm{m}+\mathrm{c}}\right)\end{array}$ & $\mathrm{R}^{2}$ \\
\hline 4,47 & 2,95 & $\mathrm{hf}_{\mathrm{m}}=3,2310^{9} \mathrm{Q}$ & 0,999 & $\mathrm{hf}_{\mathrm{m}+\mathrm{c}}=3,5810^{9} \mathrm{Q}^{1,800}$ & 0,999 \\
5,66 & 3,91 & $\mathrm{hf}_{\mathrm{m}}=8,7910^{8} \mathrm{Q}^{1,78}$ & 0,999 & $\mathrm{hf}_{\mathrm{m}+\mathrm{c}}=1,0510^{9} \mathrm{Q}^{1,790}$ & 0,999 \\
4,09 & 2,95 & $\mathrm{hf}_{\mathrm{m}}=4,6710^{9} \mathrm{Q}^{1,79}$ & 0,999 & $\mathrm{hf}_{\mathrm{m}+\mathrm{c}}=5,4510^{9} \mathrm{Q}^{1,802}$ & 0,999 \\
4,63 & 2,95 & $\mathrm{hf}_{\mathrm{m}}=2,4810^{9} \mathrm{Q}^{1,78}$ & 0,999 & $\mathrm{hf}_{\mathrm{m}+\mathrm{c}}=2,8910^{9} \mathrm{Q}^{1,794}$ & 0,999 \\
\hline
\end{tabular}

Arbitrando-se valores de vazão para as equações da Tabela 4, a perda de carga no conector foi determinada, possibilitando o ajuste de equações para a perda de carga no conector (Tabela 5).

TABELA 5. Equações de perda localizada de carga $(\mathrm{m})$ em função da vazão $\left(\mathrm{m}^{3} \mathrm{~s}^{-1}\right)$ para cada conjunto microtubo/conector. Local head losses $(\mathbf{m})$ equations as a function of the discharge $\left(\mathrm{m}^{3} \mathrm{~s}^{-1}\right)$ for each system microtube/connector.

\begin{tabular}{ccc}
\hline $\mathrm{D}_{\mathrm{m}}(\mathrm{mm})$ & $\mathrm{D}_{\mathrm{c}}(\mathrm{mm})$ & Equação de Perda Localizada de Carga no Conector \\
\hline 4,47 & 2,95 & $\mathrm{hf}_{\mathrm{c}}=4,9910^{8} \mathrm{Q}^{1,90}$ \\
5,66 & 3,91 & $\mathrm{hf}_{\mathrm{c}}=8,8310^{8} \mathrm{Q}^{2,10}$ \\
4,09 & 2,95 & $\mathrm{hf}_{\mathrm{c}}=7,1710^{9} \mathrm{Q}^{2,15}$ \\
4,63 & 2,95 & $\mathrm{hf}_{\mathrm{c}}=8,8510^{8} \mathrm{Q}^{1,96}$ \\
\hline
\end{tabular}


Na Figura 7, ilustram-se as curvas de perda de carga para os conjuntos microtubo/conector. Observa-se que o mesmo conector $\left(D_{c}=2,95 \mathrm{~mm}\right)$ teve comportamentos diferentes quanto à perda de carga quando conectado em microtubos de diâmetros diferentes, sugerindo que $h f_{c}$ depende não somente do diâmetro da peça e da velocidade média da água, mas também do diâmetro do tubo em que está inserido. MELO et al. (2000) estudaram as perdas de carga na passagem lateral em conectores de 12; 14 e $16 \mathrm{~mm}$, tendo estabelecido uma relação entre o diâmetro do tubo e diâmetro do conector. Quanto maior essa relação, maior a perda de carga, pois a perda localizada de carga resulta do choque de moléculas de água dotadas de velocidades diferentes, e quanto maior a diferença dessas velocidades, mais intensa é a turbulência.

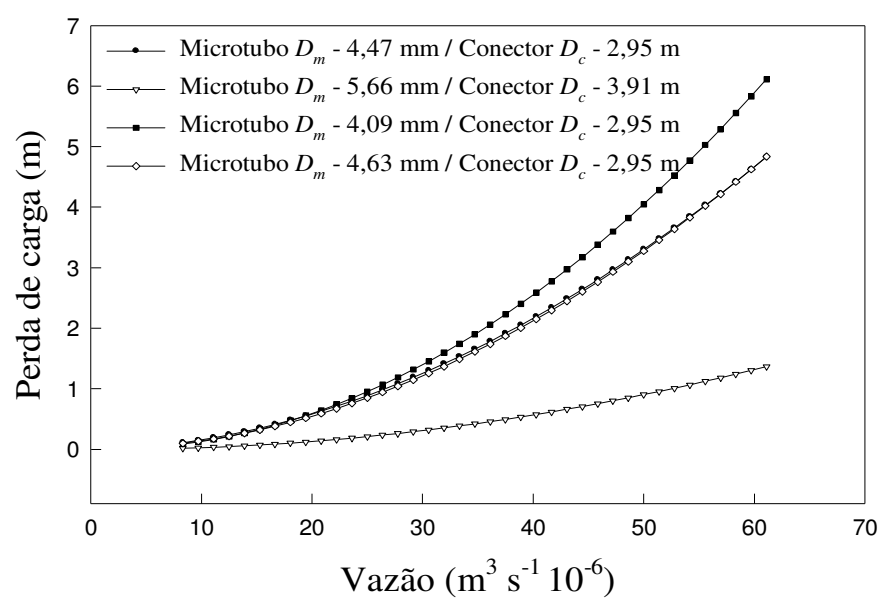

FIGURA 7. Perda de carga (m) em função da vazão $\left(\mathrm{m}^{3} \mathrm{~s}^{-1}\right)$ para cada conjunto microtubo/conector. Head loss $(\mathrm{m})$ as function of the discharge $\left(\mathrm{m}^{3} \mathrm{~s}^{-1}\right)$ for each system microtube/connector.

A perda localizada de carga é representada classicamente como uma função da carga cinética multiplicada por um coeficiente $(\mathrm{K})$, que depende da geometria da peça e do número de Reynolds. Uma modificação desse coeficiente (K') foi apresentada na eq.(10), em que o comprimento da peça foi considerado. Os coeficientes de carga cinética (K') apresentados na Tabela 6 foram ajustados para cada conjunto microtubo/conector por meio de regressão entre os valores de perda de carga unitária no conector $\left(\mathrm{J}_{\mathrm{c}}\right)$ e carga de velocidade $\left(\mathrm{V}_{\mathrm{c}}^{2} / 2 \mathrm{~g}\right)$.

TABELA 6. Coeficiente de carga cinética (K') ajustado para cada conjunto microtubo/conector. Kinetic head loss coefficient ( $K^{\prime}$ ) adjusted for each system microtube/connector.

\begin{tabular}{ccc}
\hline $\mathrm{D}_{\mathrm{m}}(\mathrm{mm})$ & $\mathrm{D}_{\mathrm{c}}(\mathrm{mm})$ & Coeficiente de Carga Cinética $\left(\mathrm{K}^{\prime}\right)$ \\
\hline 4,47 & 2,95 & 52,93 \\
5,66 & 3,91 & 47,41 \\
4,09 & 2,95 & 56,64 \\
4,63 & 2,95 & 50,62 \\
\hline
\end{tabular}

Em decorrência dos coeficientes de carga cinética ajustados ( $\left.\mathrm{K}^{\prime}\right)$, a perda localizada de carga no conector pode ser expressa pela eq.(14), em função da velocidade de escoamento no conector $\left(\mathrm{V}_{\mathrm{c}}\right)$ e do comprimento do conector $\left(\mathrm{L}_{\mathrm{c}}\right)$ :

$$
\mathrm{hf}_{\mathrm{c}}=\mathrm{K}^{\prime} \frac{\mathrm{V}_{\mathrm{c}}^{2}}{2 \mathrm{~g}} \mathrm{~L}_{\mathrm{c}}
$$

Na Figura 8, apresenta-se a variação dos coeficientes K' em função do número de Reynolds. Embora K' demonstre dependência do número de Reynolds para $\operatorname{Re}>5.000$, essa função é fraca. Considerando que a maioria dos sistemas de microaspersão trabalha em regimes turbulentos com 
Re $>5.000$, sugere-se um K' constante, que foi ajustado para todos os conjuntos microtubo/ conectores na Figura 9. BAGARELLO et al. (1997) e CARDOSO \& FRIZZONE (2007) mostraram que, para números de Reynolds superiores a $10^{4}$, o fator de carga cinética é praticamente independente de Re, e para fins de aplicação prática, pode-se considerar $K^{\prime}$ constante em determinada peça desde que o regime de escoamento seja turbulento. BAGARELLO et al. (1997) estudaram a perda de carga causada pela protrusão de emissores "on-line" e consideram o coeficiente de carga cinética independente das forças viscosas e dependente de uma relação entre a seção do tubo e a seção de escoamento onde o emissor está inserido.

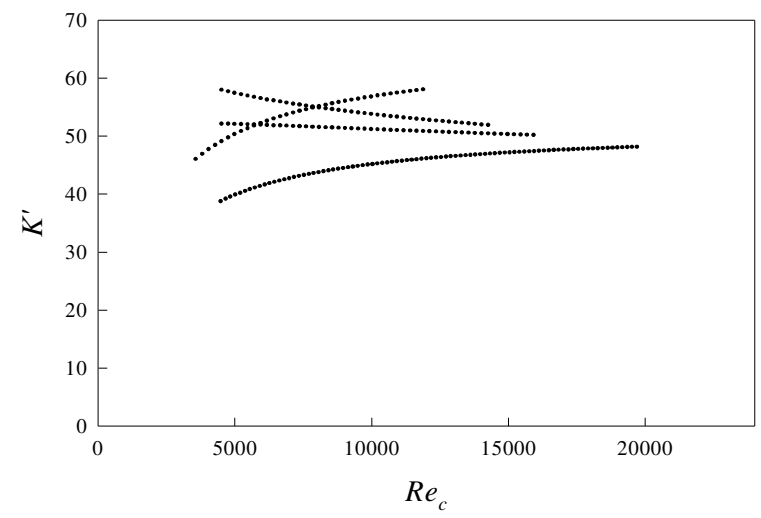

FIGURA 8. Variação do fator K' em função do número de Reynolds no conector. $K^{\prime}$ factor as a function of the Reynolds number in the connector.

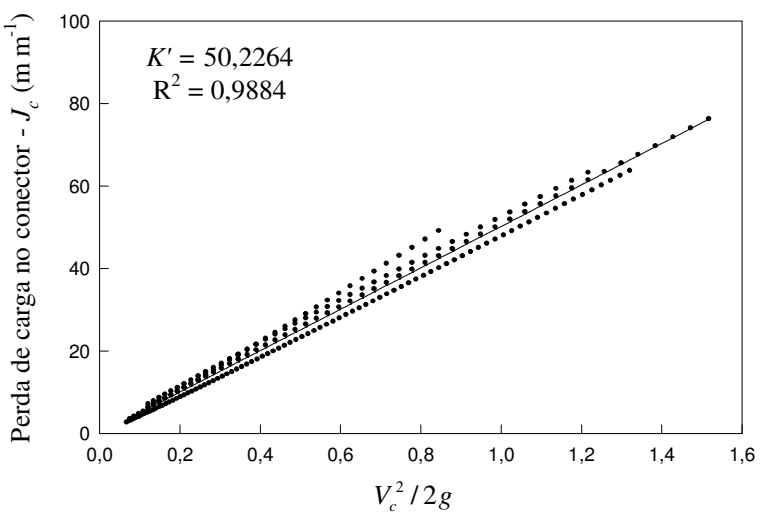

FIGURA 9. Perda localizada de carga em função da carga cinética no conector. Local head loss as function of the connector kinetic head coefficient.

Para cada valor experimental de perda de carga no conector, conhecidos os coeficientes $f$ e $K^{\prime}$, determinou-se o comprimento equivalente para cada conector por meio da eq. (12). Substituindo as variáveis conhecidas, tem-se que o comprimento equivalente é uma função dos diâmetros do microtubo, do conector e da velocidade de escoamento, conforme eq.(15):

$$
\mathrm{Le}=5,4610^{3} \mathrm{~L}_{\mathrm{c}} \frac{\mathrm{D}_{\mathrm{m}}^{5,25}}{\mathrm{D}_{\mathrm{c}}^{4}} \mathrm{~V}_{\mathrm{m}}^{0,25}
$$

HOWELL \& BARINAS (1980) ajustaram equações do tipo Le $=a Q^{b}$ para conexões de PVC e gotejadores em linhas de polietileno. Essa equação também foi utilizada por CAIXETA (1991) para expressar a perda de carga em conexões. De maneira similar, a perda de carga unitária e a perda localizada de carga variam em função de uma potência da velocidade, da mesma forma que o comprimento equivalente, podendo ser expresso pela eq.(16):

$$
\mathrm{Le}=\mathrm{aV} \mathrm{b}_{\mathrm{m}}^{\mathrm{b}}
$$

De acordo com as eqs.(15) e (16), b =0,25 e $a$ é uma função da geometria da peça descrita pela eq.(17):

$$
\mathrm{a}=5,4610^{3} \mathrm{~L}_{\mathrm{c}} \frac{\mathrm{D}_{\mathrm{m}}^{5,25}}{\mathrm{D}_{\mathrm{c}}^{4}}
$$

\section{Aplicação}

Para ilustrar o efeito da perda de carga provocada pelo microtubo e pelo conector, uma linha lateral em nível foi dimensionada pelo procedimento trecho a trecho, iniciando-se pelo último emissor. O critério de uniformidade adotado foi uma variação máxima de vazão de 10,3\% entre os emissores localizados nas extremidades da linha. O microaspersor utilizado possui pressão de serviço de $146 \mathrm{kPa}(15 \mathrm{~m})$, vazão nominal de $31 \mathrm{~L} \mathrm{~h}^{-1}$ e a equação característica: $\mathrm{q}_{\left(\mathrm{Lh}^{-1}\right)}=7,28 \mathrm{H}_{(\mathrm{m})}^{0,535}$. 
$\mathrm{O}$ espaçamento entre emissores foi definido em $4 \mathrm{~m}$ com microtubo de $1,5 \mathrm{~m}$ de comprimento e 4,09 mm de diâmetro. Para esse microtubo, utilizou-se o conector de 2,95 $\mathrm{mm}$ de diâmetro inserido em uma linha lateral de $17,4 \mathrm{~mm}$ de diâmetro. A pressão máxima na entrada da lateral foi assumida como $166,6 \mathrm{kPa}(17 \mathrm{~m})$, e a pressão mínima no final da lateral de $139,16 \mathrm{kPa}(14,2 \mathrm{~m})$, com $\mathrm{H}_{\mathrm{var}}$ máximo na lateral de $16,5 \%$.

A partir dos limites de pressão, a linha lateral foi dimensionada considerando-se apenas a perda distribuída de carga, calculada pela equação de Darcy-Weisbach. O fator de atrito foi calculado pela equação de Blasius com coeficiente $c$ igual a 0,300 , sugerido por CARDOSO et al. (2008), para tubos de polietileno de 17,4 mm de diâmetro.

Uma segunda etapa do dimensionamento consistiu em verificar para essa linha quais seriam as variações de vazão e de pressão entre os emissores das extremidades caso fossem consideradas as perdas de carga localizadas causadas pela passagem direta por meio do conector, pela passagem lateral e pelas perdas contínuas no microtubo. A perda de carga provocada pela passagem direta foi calculada com base na equação do índice de obstrução (IO), proposta por CARDOSO \& FRIZZONE (2007).

Na Figura 10, são apresentados os valores de $\mathrm{q}_{\mathrm{var}}$ e $\mathrm{H}_{\mathrm{var}}$ entre os emissores nos extremos da lateral, para o dimensionamento que considera apenas a perda distribuída de carga na linha lateral ( hf no microtubo mais conector $=0$ ) e para os dimensionamentos que consideram perda de carga na passagem direta e diferentes valores de perdas de carga no conector mais microtubo. Verifica-se que, ao se considerar apenas a perda distribuída de carga na linha lateral, dimensiona-se uma tubulação de $104 \mathrm{~m}$ de comprimento máximo com $\mathrm{q}_{\mathrm{var}}$ de $8 \%$ e $\mathrm{H}_{\mathrm{var}}$ de $14,4 \%$. Para esse mesmo comprimento de lateral, ao serem consideradas as perdas de carga na passagem direta, na passagem lateral pelo conector e no microtubo, as variações de vazão e pressão são aumentadas. Para a perda de carga no conector mais microtubo igual a $0,420 \mathrm{~m}$, as variações de vazão e pressão foram maiores $(8,50 \%$ e $15,3 \%$, respectivamente), porém não violaram os critérios estabelecidos. Além desse ponto, as variações de vazão e pressão cresceram linearmente, passando a violar os critérios de uniformidade de projeto estabelecidos para perdas de carga $(0,680 \mathrm{~m})$.

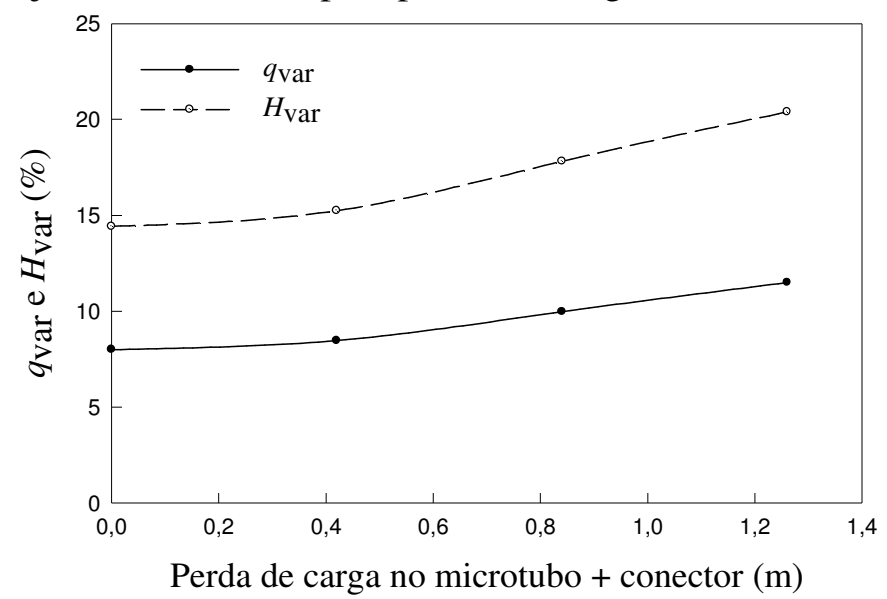

FIGURA 10. Variação de vazão e de pressão nos emissores localizados nos extremos de uma linha lateral de $104 \mathrm{~m}$ de comprimento, em função da perda de carga no microtubo mais conector. Discharge and pressure head variation in emitters located in extremes side of the $104 \mathrm{~m}$ length line, as a function of the head loss in microtube added connector.

\section{CONCLUSÕES}

$\mathrm{O}$ fator de atrito $\mathrm{f}$ da equação universal de perda de carga para os microtubos estudados, com diâmetros internos de 4,47; 5,66; 4,09 e 4,63 mm, pode ser estimado pela equação de Blasius com $\mathrm{c}=0,290$ e $\mathrm{m}=0,25$, para fins de cálculo da perda contínua de carga. 
A perda localizada de carga na passagem lateral do conector pode ser expressa em função do comprimento do conector e da carga cinética nessa passagem corrigida por um coeficiente $\mathrm{K}$ ' igual a 50,2 para qualquer conjunto microtubo/conector.

O coeficiente de carga cinética (K') variou pouco em função do número de Reynolds, podendo ser considerado constante, para aplicações práticas, a partir de $\operatorname{Re}>5.000$, para cada conjunto microtubo/conector estudado.

Em termos de comprimento equivalente, a perda localizada de carga no conector pode ser expressa em função de uma potência $(0,25)$ da velocidade de escoamento no microtubo, corrigida por um coeficiente $a$, que é diretamente proporcional ao comprimento do conector e a uma potência do diâmetro do microtubo $(5,25)$, e inversamente proporcional a uma potência (4) do diâmetro do conector.

As variações de vazão e de pressão entre os emissores situados nos extremos da linha lateral mostraram-se sensíveis à perda de carga na passagem lateral pelo conector mais a perda de carga no microtubo.

\section{AGRADECIMENTOS}

Ao Ministério da Ciência e Tecnologia (MCT), ao Conselho Nacional de Desenvolvimento Científico e Tecnológico $(\mathrm{CNPq})$ e à Fundação de Amparo à Pesquisa do Estado de São Paulo (FAPESP), pelo apoio financeiro a esta pesquisa, por meio do Instituto Nacional de Ciência e Tecnologia em Engenharia da Irrigação (INCTEI).

\section{REFERÊNCIAS}

ALVES, P.R.V.; PORTO, R. Coeficiente geométrico para estimativa da perda de carga localizada em linhas laterais de irrigação por gotejamento. Engenharia Agrícola, Jaboticabal, v.22, n.1, p.51$59,2002$.

BAGARELLO, V.; FERRO, V.; PROVENZANO, G.; PUMO, D. Experimental study on flow resistance law for small-diameter plastic pipes. Journal of Irrigation and Drainage Engineering, New York, v.121, n.5, p.313-316, 1995.

BAGARELLO, V.; FERRO, V.; PROVENZANO, G.; PUMO, D. Evaluating pressure losses in drip-irrigation lines. Journal of Irrigation and Drainage Engineering, New York, v.123, n.1, p.1-7, 1997.

BERNUTH, R.D. Von. Simple and accurate friction loss equation for plastic pipe. Journal of Irrigation and Drainage Engineering, New York, v.116, n.2, p.294-298, 1990.

CAIXETA, A.V. Perda de carga em tubos e conexões de PVC utilizados em sistemas portáteis de irrigação por aspersão. 1991. 127 f. Dissertação (Mestrado em Irrigação e Drenagem) - Escola Superior de Agricultura “Luiz de Queiroz”, Universidade de São Paulo, Piracicaba, 1991.

CARDOSO, G.G.G.; FRIZZONE, J.A. Perda de carga em linhas laterais de microirrigação. Revista Brasileira de Engenharia de Biossistemas, Campinas, v.1, n.2, p.147-162, 2007.

CARDOSO, G.G.G.; FRIZZONE, J.A.; REZENDE, R. Fator de atrito em tubos de polietileno de pequenos diâmetros. Acta Scientiarum Agronomy, Maringá, v.30, n.3, p.299-305, 2008.

FRIZZONE, J.A.; VIEIRA, A.T.; PAZ, V.P.S. Caracterização hidráulica de um tubo gotejador. Revista Brasileira de Engenharia Agrícola e Ambiental, Campina grande, v.2, n.3, p.278-283, 1998.

HOWELL, T.A.; BARINAS, F.A. Pressure loss across trickle irrigation fitting and emitters. Transactions of the ASAE, St. Joseph, v.23, n.4, p.67-72, 1980. 
JUANA, L.; RODRIGUES-SINOBAS, L.; LOSADA, A. Determining minor head losses in drip irrigation laterals. I: Methodology. Journal of Irrigation and Drainage Engineering, New York, v.128, n.6, p.376-384, 2002.

MELO, J.V.; SILVA, E.L.; FARIA, M.A. Perda de carga em conectores utilizados em sistemas de irrigação localizada. Ciência Agrotécnica, Lavras, v.24, (Edição especial), p.205-214, 2000.

PORTO, R.M. Hidráulica básica. São Carlos: USP, EESC, 1998. 540 p.

PROVENZANO, G.; PUMO, D. Experimental analysis of local pressure losses for microirrigation laterals. Journal of Irrigation and Drainage Engineering, New York, v.130, n.4, p.318-324, 2004.

RETTORE NETO, O.; FRIZZONE, J.A.; MIRANDA, J.H.; BOTREL, T.A. Perda de carga localizada em emissores não coaxiais integrados a tubos de polietileno. Engenharia Agrícola, Jaboticabal, v.29, n.1, p.28-39, 2009.

ROMEO, E.; ROYO, C.; MONZÓN, A. Improved explicit equation for estimation of the friction factor in rouge and smooth pipes. Chemical Engineering Journal, Lausanne, v.86, n.3, p.369-374, 2002.

SONNAD, J.R.; GOUDAR, C.T. Turbulent flow friction factor calculation using a mathematically exact alternative to the Colebrook-White equation. Journal of Hydraulics Engineering, New York, v.132, n.8, p.863-867, 2006.

VILELA, L.A.A.; SOCCOL, O.J.; GERVÁZIO, E.S.; FRIZZONE, J.A.; BOTREL, T.A. Alteração no diâmetro de tubos de polietileno submetidos a diferentes pressões. Revista Brasileira de Engenharia Agrícola e Ambiental, Campina Grande, v.7, n.1, p.182-185, 2003.

YILDIRIM, G. An assessment of hydraulic design of trickle laterals considering effect of minor losses. Journal of Irrigation and Drainage Engineering, New York, v.56, n.4, p.399-421, 1997.

YITAYEW, M. Simplified method for sizing laterals with two or more diameters. Journal of Irrigation and Drainage Engineering, New York, v.135, n.1, p.111-114, 2009.

YOO, H.; SINGH, V.P. Two methods for the computation of commercial pipe friction factors. Journal of Hydraulics Engineering, New York, v.131, n.8, p.694-704, 2005. 